\title{
MARE ISLAND FLEXIBLE MANUFACTURING WORKSTATION
}

\author{
Kang Lee, Charles Yang
}

National Bureau of Standards,

Gaithersburg, MD 20899

ABSTRACT

The Mare Island Flexible Manufacturing Workstation (MIFMW) is a fully automated flexible turning workstation being developed at the National Bureau of Standards (NBS). It is designed to produce parts, unattended, for extended periods of time. It has many capabilities and sophisticated features which include hierarchical control architecture, in-process gaging, tool setting, tool condition monitoring, automated buffer storage of workpieces and supplies, robotic workpiece loading and unloading, and robotic changing of tools, chuck jaws, and gripper fingers. Its scheduled completion date is the fall of 1988, at which time the workstation will begin production at the Mare Island Naval Shipyard machine shop near San Francisco. When completed, the MIFMW is expected to be one of the first small-batch flexible manufacturing systems in the U.S. capable of running unattended for 24 hours a day, five days a week.

\section{INTRODUCTION}

The MIFMW is a prototype production workstation that is being developed to meet Department of Defense needs and to study workstation integration in a production environment. The project is a joint research venture between the U.S. Navy, Mare Island Naval Shipyard, and NBS to automate the manufacture of Resonance Inhibiting Sound Isolating Connectors (RISIC), which are critical components used in the maintenance of nuclear submarines. Many of the technologies involved in the development of this workstation are directly transferred from the research done in the areas of Computer Integrated Manufacturing (CIM) and Flexible Manufacturing Systems (FMS) at the Automated Manufacturing Research Facility (AMRF) at NBS [1]. In particular, numerous techniques are derived from work done at the Turning Workstation in the AMRF [2]. Other technologies are currently being developed at the Automated Production Technology Division at NBS. The research and development efforts are supported by the Navy Manufacturing Technology program and NBS, along with cooperation from the industrial sector.

\section{MOTIVATION FOR AUTOMATION}

The Mare Island Naval shipyard machine shop produces a wide variety of ship parts in small-lot sizes on a "just-in-time" basis: that is, a part is produced when it is ordered and no stock is kept. This type of operation in a manually operated machine shop environment inhibits high productivity. As a matter of fact, it presently takes an average of 17 hours to produce a RISIC part component. Through automation the average production time of a RISIC part can be reduced to 3 hours. However, in this case a "just-in-time" operation is very difficult to automate due to the wide variety of parts that must be produced. Currently, most automated high-volume manufacturing work-cells execute a fixed sequence of operations repeatedly to produce a large number of a particular part. This type of workstation is "inflexible" in the sense that in order to use the same workstation to produce a different part, extensive off-line reprograming of the devices within the workstation is required. Clearly, a more "flexible" manufacturing workstation that could machine a wide variety of parts without reprogramming is more desirable.

\section{DESIGN CONSIDERATION}

The goal of the MIFMW project is to develop an effective workstation design to efficiently automate the manufacturing of the families of RISIC parts. The RISIC is broken down into four families, with ten sizes of geometrically dissimilar parts within each family. That means the MIFMW must be able to manufacture forty different parts. The workpieces vary in diameter from 1.25 inches to 11 inches, and the largest workpiece weighs 150 pounds. The machining of every part within the RISIC family in small mixed batches without human intervention brings about a spectrum of challenging engineering problems that must be solved. The continuous unattended production of mixed batches dictates that the MIFMW must perform the following functions:

1. Accept a random sequence of non-identical workpieces and provide the machining in a continuous, three-shift operation.

2. Regroup the tooling on the turning center tool 
turret in between machining of different parts.

3. Change the workholding device for machining of different parts, if needed.

4. Maintain quality control and detect malfunctions through the use of sensors and other analys is techniques.

5. Coordinate control of machine tool and handling devices for workpieces and tooling.

The following approaches are being incorporated in the workstation to achieve the defined functions:

1. A robot manipulator with proper load-handling capacity to automate workpiece loading and unloading.

2. An adaptable workholding system with interchangeable jaws for the turning center to deal with the wide range of part diameters.

3. Interchangeable live tooling as well as standard tooling to change worn-out or defective tools.

4. A flexible robot end-effector with interchangeable fingers to handle the workpieces and the tooling.

5. A buffer system to support storage and retrieval of workpieces, tooling, and gripper fingers.

6. An in-process gaging system and a tool-setting station to maintain part integrity.

7. A computer with hierarchical control architecture to supervise and control the complex manufacturing operation.

\section{WORKSTATION DESCRIPTION}

The MIFMW is composed of a CNC turning center that supports turning and machining operations, an opengantry robot with a flexible end-effector system that performs workpiece, tooling, and chuck jaw handling, a random-access buffer system that provides storage and retrieval of supplies, a malfunction detector that monitors tool condition, and a workstation controller that coordinates workstation activities. A hierarchical control structure is employed within the MIFMW. The workstation controller and the workstation devices communicate through serial communication links. The workstation controller issues high-level commands to the devices which return their status to the workstation controller indicating their state of execution. All servoing and other lower level control are handled by the individual device controllers. Some of the devices obtain data information from the workstation database to enable them to perform their tasks. A MIFMW overview is shown in figure 1 , and a MIFMW control architecture is shown in figure 2 .
The devices of the MIFMW includes a Warner Swasey WSC-12 turning center $* *$, a Westinghouse Unimate 6000 robot, a Kardex Automatic Storage/Retrieval System (AS/RS), an NBS-designed robot gripper mounted on the robot's wrist, an NBS-designed malfunction detector, and a microcomputer-based workstation controller. The turning center is used to machine the parts, and is specially equipped with live tooling stations, a tool-setting station, and a Renishaw touch probe used for gaging. The Westinghouse robot was chosen for its $300 \mathrm{lb}$ payload capacity, and Cartesian coordinate and open gantry design, which simplify loading operations with the turning center. In addition, the robot's functions can be easily expanded to serve another machining system by adding a rotary waist between the robot arm and the gantry. The AS/RS buffer system is a two-stack vertical structure with tray elevators that move trays from any shelf to a presentation area that is robot-accessible. The NBS gripper is a microprocessor-based electromechanical device capable of fine positioning and force control. Another important feature of the gripper is its interchangeable fingers which enable the gripper to grasp a variety of objects. The NBS malfunction detector is used to monitor machining vibrations during machining operations. It functions as a tool condition monitor as well as a safety device.

The workstation controller is a software application written in PLM86, and is based on Intel's iRMX86 operating system. The software runs on an Intel System 310/286 microcomputer with a fortymegabyte hard disk that serves as the workstation database. In addition to controlling workstation devices, the workstation controller also serves as a human interface, enabling the operator to order part batches, load workpieces into the AS/RS, examine workstation status, and perform diagnostics. The workstation controller maintains the workstation database and provides inventory and tool condition information as well. A system block diagram of the workstation controller is shown in figure 3 .

\section{ENABLING TECHNOLOGIES}

A number of technologies previously developed at the AMRF, in addition to those developed specifically for this project, are used to achieve the workstation's objective. Numerous methodologies developed by others in FMS research were also considered, and some were implemented. The technologies include automated workpiece and tool handiing, workholding device changing, live tooling, quality control, and hierarchical control.

\section{AUTOMATED WORKPIECE AND TOOL HANDLING}

$A$ number of workpiece and tool handling strategies were considered in the development of MIFMW. A popular approach in workpiece loading/unloading is to use a dedicated workpiece handling robot in conjunction with a workpiece buffering device that feeds it [3]. In this case, typical buffering systems include chutes, conveyors, and carousel feeders with workpiece holding pallets. The 
dedicated robot/buffer concept is employed in tool changing as well. A tool changer is coupled with tool buffering devices such as magazines, drums, and chains $[3,4,5]$. The manipulators and buffering devices are often extensions of the turning center and are typically controlled by the turning center controller [5].

The dedicated robot/buffer methods are very effective for parts with a narrow range of sizes and geometries. However, there are no automated turning centers of this type in the market today that have enough flexibility to produce the families of RISIC parts.

In addition, these dedicated buffering devices typically do not have large storage capacity and consume too much floor space. To modify an existing turning center with a dedicated robot or to develop a new one specifically for dealing with the material handling needs of this project would be cost prohibitive and would not be practical. Therefore, the dedicated robot/buffer approach is not feasible for the development of MIFMW.

Another strategy used to solve the workpiece and tool handling problem is to use a single robot to perform all the material handling required within the workstation. This approach eliminates the need for specifically dedicated manipulators. It also eliminates the need for dedicated buffering devices and allows the use of a single, more generic buffer system, that can store a variety of materials and supplies. More importantly, this reduces the amount of floor space required by the workstation. However, this approach requires an end-effector system capable of handling objects with varying geonetries.

The researchers in the AMRF have extensive experience on the design of flexible robot endeffectors or robot grippers $[2,16]$, therefore the single robot approach was chosen to implement the design of the MIFMW. The Westinghouse Unimate 6000 robot is used to perform tool and chuck jaw changing, as well as workpiece loading and unloading. A flexible robot gripper system is adapted to facilitate these functions. The gripper incorporates a dove-tail design that enables a variety of gripper fingers to slide onto it. This allows the gripper to grasp a variety of objects by utilizing fingers designed for the particular geometries and weights. The servo-controlled gripper also incorporates a number of sophisticated features such as position control, force control, self-calibration through force mapping to account for systematic errors such as friction, and a highlevel controller $[6,16]$. The gripper can also be used to identify workpiece size by measuring its diameter using position feedback.

Because a single robot capable of adaptable object manipulation is employed, a flexible buffer system can be used to support it. The Kardex AS/RS is used to store workpieces, tools, chuck jaws, and gripper fingers. Its design is based on the concept of a database system. This means the AS/RS is treated as a random-access device which receives object-oriented commands from the workstation controller and utilizes the workstation database to determine the location of objects and retrieve them for output [7].

\section{WORKHOLDING DEVICE CHANGING}

There are no workholding devices on the market today capable of fixturing turning workpieces whose diameters range from 1.25 to 11 inches without some sort of reconfiguration. Thus, the process of workholding device changing must be automated. A chuck is used as the workholding device in the MIFMW, and a number of replacement strategies were investigated. Interchangeable chucks and interchangeable chuck jaws have been implemented in other systems $[8,9]$. In the MIFMW design, approximately 21 different chuck sizes would be needed to cover the entire range of part diameters; the use of interchangeable chucks is prohibitive due to their cost, weight, and the use of a rather large and dedicated robot to implement the system. Hence, the alternative automated chuck jaw changing system is implemented.

However, automated chuck-jaw changing is a fairly complicated process, requiring robotic assembly of jaws onto a loading pallet which is mated with an empty chuck to load the jaws $[8,9]$. Conversely, an empty pallet is mated with a chuck loaded with jaws to remove them from the chuck onto the pallet. The cost of the chuck-jaw changing system is further decreased by using only two pallets instead of using a pallet for each set of chuck jaws. This means one pallet set is used to unload chuck jaws from the chuck, while the other one is used to load chuck jaws onto the chuck. Of course, operation in this manner requires the robot to assemble chuck jaws onto the pallet and vice versa. The process of chuck jaw assembly and disassembly, as well as the coupling of the chuck with the loading pallets, are both achieved by the robot in conjunction with the flexible gripper system.

\section{LIVE TOOLING}

All RISIC parts have features that require machining, off-axis drilling and tapping, and radial drilling and tapping, which cannot be done by most turning centers. A number of advantages are gained by doing these operations within the turning center. Firstly, accuracy degradation due to refixturing is avoided. Otherwise, tedious and costly re-fixturing of parts would be required if two (or more) different types of machines are used to make the parts. Secondly, time-consuming tool setting between machines can be eliminated as well. The MIFMW turning center is equipped with live tooling stations in both radial and axial orientations to perform the necessary machining operations. The live tooling spindles on the tool turret are being re-designed so that live tooling can be robotically changed.

\section{OUALITY CONTROL}

A number of quality control techniques are implemented in the MIFMW. Tool condition monitoring is one useful technique for quality control, since machining a part with worn tools degrades part accuracy. There are two general strategies of tool 
condition monitoring implemented today. One strategy is to use the time-based approach, where the life span of a tool is previously determined from observation, and the amount of time a tool is used is the single criterion for determining tool wear [3]. Another strategy is to use sensory techniques such as vibration and force measurement $[10,11]$. In the MIFMW, both techniques are used to optimize tool utilization. A tool may be replaced if the vibrational threshold is exceeded prior to projected time threshold, or vice versa. Both time-based and sensor-based tool condition analyses are performed by the malfunction detector, which engages the workstation database to achieve this function.

Tool setting is an essential technique for quality control. When a tool on the tool turret is replaced, the absolute position of the new tool tip is not known, and this uncertainty diminishes the accuracy of parts machined with it. Therefore a new tool must be "set" before it can be used. The absolute location of the tool tip is measured by the tool-setting station, and the tool offset registers in the turning center control are updated to compensate for the change in location [12].

In-process part gaging is another useful technique, as it can be used to compensate for errors caused by changing environmental conditions and gradual tool wear. Since a RISIC part may require up to two hours of machine time, errors caused by changing environmental conditions such as thermal gradients at machining time can become especially prominent. One strategy considered for gaging is to gauge a part after it is completely machined to obtain dimensional data for error compensation for the next part $[3,11]$. This method has the disadvantage in that the compensation is performed after the fact, which can be wasteful of time and material in a small-batch environment. The more useful approach to be adapted in the MIFMW is to gauge the part prior to the completion of a feature and compensate for errors before the last cuts are made [11].

\section{HIERARCHICAL CONTROL}

Several different work-cell configurations were investigated in determining the MIFMW system architecture. One popular approach is to use a turning center that is equipped with material handling devices such as a workpiece loader and buffer $[3,5]$. In this case, all the devices are controlled by the turning center control through NC programming. Another approach is to mate devices to the turning center $[3,5]$. In this case, the device control is performed by the individual controllers, and their executions are synchronized with the execution of the turning center through digital I/O communication lines. Although these configurations are effective for a small variety of parts, current turning centers that have these configurations do not have enough hardware and software flexibility to meet the requirements of this project.

In both approaches above, highly sophisticated and specialized turning center control would be required, since it would have to perform control at every level, from the user front-end to servo control. The turning center control would also have to plan the sequence of device executions, since it is difficult to hard code the sequence due to varying tooling and workholding configurations that would be required for a large number of different parts. Interfacing additional devices to the workstation would also be hampered, since turning centers typically do not provide a large number of sophisticated interfaces. To meet these needs would require an enormous amount of development and special modifications, making this approach impractical for this project.

Another approach is to employ a hierarchical architecture within the workstation $[13,14]$. In this case, a workstation controller coordinates the activities of the devices in the workstation by way of command and status protocols. Communications are established only between levels and none are allowed across the same level. This approach eliminates the need for highly specialized turning center control, as well as complicated hand-shaking between the devices, since the workstation controller would synchronize device executions. The workstation controller can also perform front-end operations such as process plan generation and inventory control.

A hierarchical control structure previously developed at the AMRF is implemented in the MIFMW. The workstation controller software runs on the iRMX86, an event-driven, multi-tasking operating system that facilitates real-time communication with workstation devices. In addition to orchestrating the device executions in the workstation, the workstation controller also functions as a human interface, enabling the operator to order part batches, load workpieces into the AS/RS, examine workstation status, and perform diagnostics. The workstation controller maintains the workstation database and provides inventory and tool condition information as well.

Workstation control is achieved by the Process Plan Generator (PPG) and the Control Module of the workstation controller. The devices within the workstation execute actions in appropriate sequence to perform a specific job. This sequence of events is manifested in a "process plan", which can be decomposed into several levels of operational data. The PPG provides operational data to the control module which executes the data and sends the commands to the workstation devices to perform the task. For each batch of parts ordered, the PPG generates a "setup" plan and a "make batch" plan. The control module schedules the workstation devices to execute setup prior to making a batch.

The PPG employs task decomposition concepts previously developed at the AMRF $[2,15]$. It decomposes the job that is to be executed into three levels of operational data, from generic to specific description. The top level is the job level. A job level work element is the most generic representation of the $j$ ob to be performed. MAKE_BATCH and SETUP are typical job elements. The next level of decomposition is the task level. Each job level work element is decomposed into a set of 
more specific task level work elements. LOAD_BLANK, UNLOAD_PART are typical task elements. Each task level work element is further decomposed into a set of device level work elements, with the device level at the lowest level of decomposition. Each device element contains a command that is to be sent to a specific workstation device. A task decomposition graph is shown in figure 4.

The PPG generates all the operational data associated with a job immediately prior to the job's execution. For each job, the PPG generates a task level operation sheet, which contains a set of task level work elements. For each task element, the PPG generates a device level operation sheet, which contains a set of device commands. Each task and device element has a step number and a set of precedence numbers that are used to determine when the element should execute. The precedence graph of a typical task level operation is shown in figure 5 .

The actual elements in a task or device level operation sheet is dependent upon some of the attributes of the part that is to be produced, and the current configuration of the workstation. Relevant part attributes include part diameter and length, tools and jaws to be used to machine it, machining vibration signature, and the NC program required. Relevant current configuration data include the set of jaws loaded on the chuck, tool turret map, NC program selected in the turning center, and the set of fingers mounted on the gripper. The above data are stored in tables in the workstation database. The PPG uses this data to generate the appropriate operation sheets. A typical part resource and attribute data table is shown in figure 6 and a typical workstation configuration table is shown in figure 7.

The Control Module has a hierarchical structure, as shown in figure 8 . The job level controller executes a job element and receives a status signal from the task level controller regarding the job's execution. The task level controller executes each element in the task level operation sheet and receives a status signal regarding a task element's execution from the device level controller. The device level controller executes each element in a device level operation sheet by sending it to the appropriate workstation device. Each device updates the device level controller on its state of execution. Each level's controller executes the elements in sequence as prescribed by the step number and the set of precedent numbers associated with each element on the operation sheets.

\section{SUMMARY}

The Mare Island Flexible Manufacturing Workstation is being developed in the Automated Manufacturing Research Facility at the National Bureau of Standards. The workstation features hierarchical control architecture, combined machining and turning operations in one machine, adaptable workholding, flexible robot end-effector, robotic changing of tools, chuck jaws, and gripper fingers, robotic workpiece loading and unloading, and automated buffer storage of supplies. Quality control in the workstation is achieved by using inprocess part gaging, tool setting, and tool condition monitoring. When completed, the workstation is expected to be one of the first class of small-batch flexible manufacturing systems in the United States capable of continuous unattended production.

\section{ACKNOWLEDGEMENT}

The continuous support of the Navy Manufacturing Technology program to the Automated Manufacturing Research Facility at the National Bureau of Standards is essential to the success of the joint development of the Mare Island Flexible Manufacturing Workstation with the Navy shipyard, the transfer of technologies to other Naval facilities, and other research activities in the AMRF.

** Certain commercial equipment, instruments, or materials are identified in this paper in order to adequately specify the experimental setup and workstation capability. Such identification does not imply recommendation or endorsement by the National Bureau of Standards, nor does it imply that the materials or equipment identified are necessarily the best available for the purpose or the only equipment that could be used.

\section{REFERENCES}

1. Simpson, J.A., Hocken, R.J., Albus, J.S., "The Automated Manufacturing Research Facility of the National Bureau of Standards", Journal of Manufacturing systems. Society of Manufacturing Engineers, 1982, Volume 1.

2. Lee, K.B., Donmez, M.A., Yang, C., "An Automated Turning Workstation in The AMRF", Proceedings of The Third Biennial International Machine Tool Technical Conference, Chicago, IL, September, 1986

3. Mason, F., Freeman, N.B., "Turning Centers Come of Age", American Machinist Special Report 773, February, 1985

4. Carter, N., "The Application of a Flexible Tooling System in a Flexible Manufacturing System," Robotica, 1985 , volume 3 , pp 221228 .

5. Tlusty, J., "Unmanned Machining Centers", Proceedings of The 1982 Canadian Conference on Industrial Computer Systems, McMaster University, Canada, May, 1982.

6. Lee, K.B., Li, W., "End-effector Controller for Automated Manufacturing," Fourth Biennial International Manufacturing Technology Conference, Chicago, IL, September 7-14, 1988. 
7. Fishman, D., Scott, H., Bunch, R., "Integration of Material Buffering Devices in an Automated Factory", Center for Manufacturing Engineering, National Bureau of Standards, Washington DC, 20234, October, 1986.

8. Wick, C., "Advances in Power Chucks", Manufacturing Engineering, February, 1987.

9. Steger, H.J., Antoni, H., "Modern Technology Workholding for FMS and Just-In-Time Manufacturing Environments," Proceedings of The Third Biennial International Machine Tool Technical Conference, Chicago, IL, September, 1986.

10. Powell, J.W., Cosic, J.E., Herko, F.M., Kline, W.A., Mayer, J.E., "Cutting Tool Sensors", Proceedings of The National Machine Tool Builders' Association Second Blennial International Machine Tool Technical Conference, September, 1984.

11. Tlusty, J., Andrews, G.C., "A Critical Review of Sensors for Unmanned Machining," Annals of CIRP, volume 32, number 2, 1983.

12. Peris, J.P., "Development of an Automated Tool Setting System for NC Turning Centers," to be published.

13. Albus, J.S., Barbara, A.J., Nage1, R.N., "Theory and Practice of Hierarchical Control", Proceedings of The 23rd IEEE Computer Society International Conference, September, 1981.

14. Bloom, H., "Hierarchical Control in an Automated Manufacturing Facility", Second Annual Robotics Conference: Robotics - Beyond Human Reach, University of Alabama, Huntsvil1e, AL, Apri1, 1982.

15. Brown, P.F., Mclean, C.R., "Interactive Process Planning in the Automated Manufacturing Research Facility", 1986 American Society of Mechanical Engineers Winter Annual Meeting, Anaheim, GA, December, 1986

16. Ousterhout, K.B., "A Robot End Effector Designed for Flexible Manufacturing Systems", Society of Manufacturing Engineers Conference on Mechanical Design and Sensor Integration, Cincinnati, Ohio, September 21-22, 1987. 

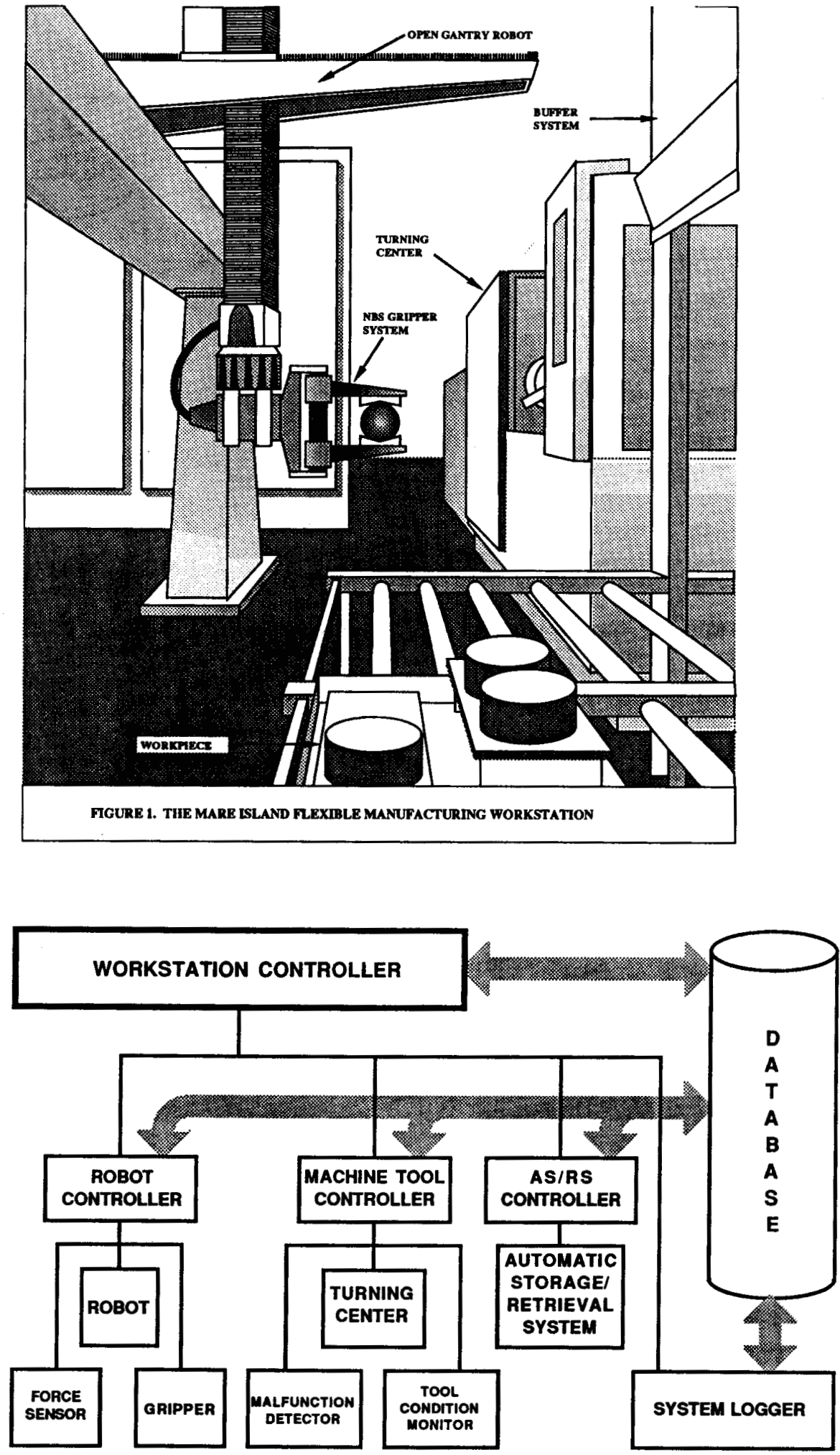

FIGURE 2: MIFMW SYSTEM ARCHITECTURE 


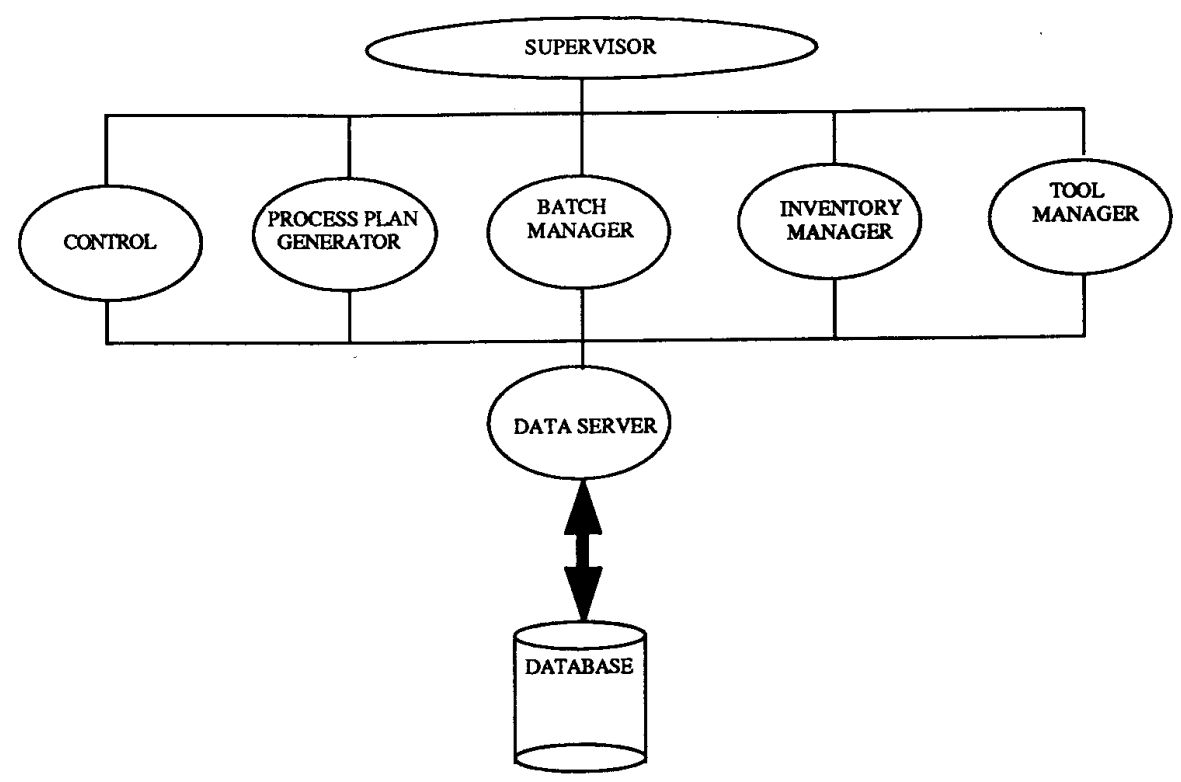

FIGURE 3: MIFMW WORKSTATION CONTROLLER SYSTEM DIAGRAM

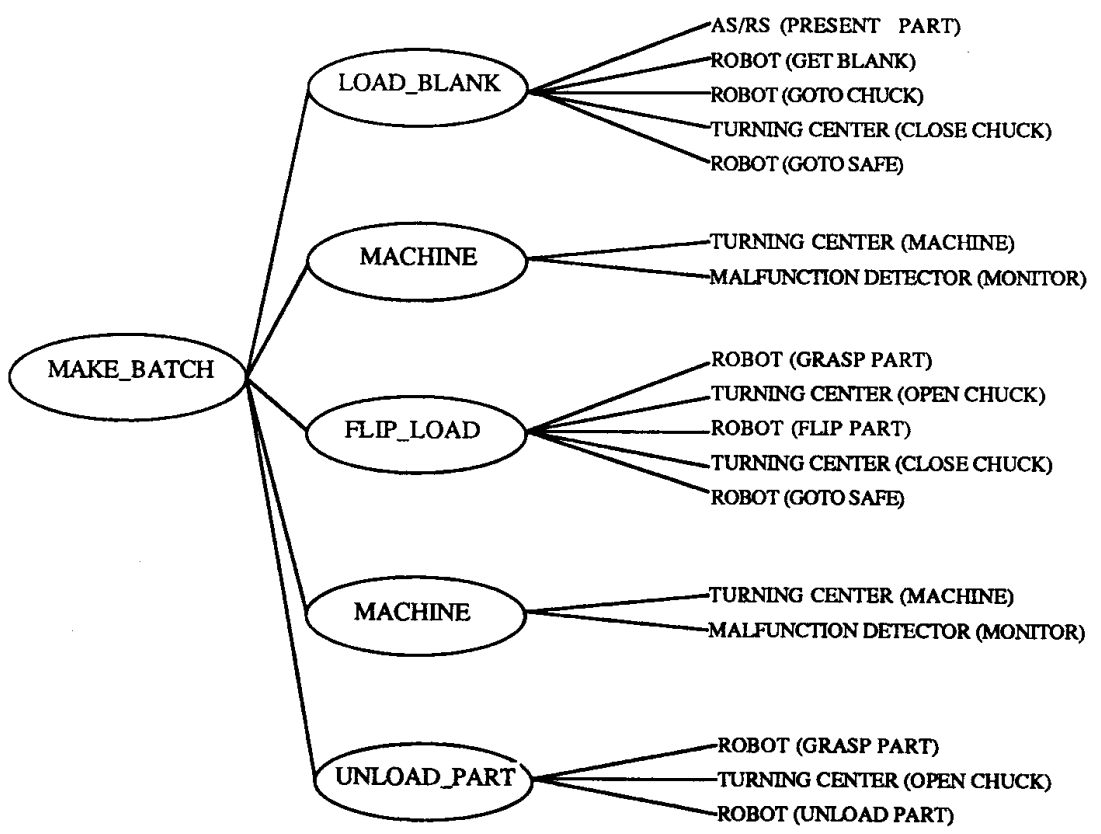

FIGURE 4: TASK DECOMPOSITION GRAPH 


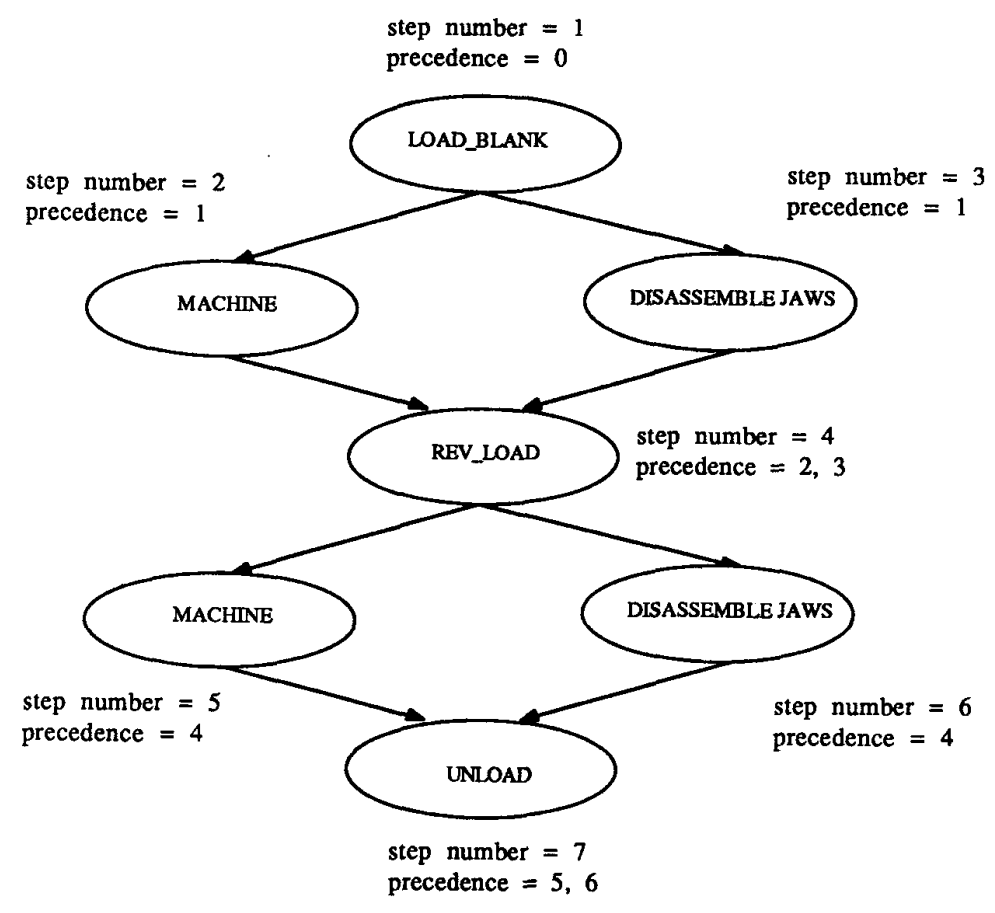

FIGURE 5: TYPICAL PRECEDENCE GRAPH

\begin{tabular}{|c|c|c|c|c|c|c|c|}
\hline part id & dia & length & NC programs & tool map 1 & tool map 2 & jaws 1 & jaws 2 \\
\hline \$P004 & 9.0 & 2.5 & $\begin{array}{c}\text { FLANGE1(4) } \\
\text { FLANGE2(4) } \\
\ldots\end{array}$ & $\begin{array}{c}\text { ST002 } \\
\text { ST005 } \\
\ldots\end{array}$ & $\begin{array}{l}\text { \$T018 } \\
\text { \$T021 }\end{array}$ & \$N003 & $\$ 012$ \\
\hline \$P005 & 9.0 & 4.5 & $\begin{array}{l}\text { BODY1(4) } \\
\text { BODY2(4) }\end{array}$ & $\begin{array}{l}\text { \$TO11 } \\
\text { ST012 }\end{array}$ & $\begin{array}{l}\text { \$TO21 } \\
\text { \$TO22 }\end{array}$ & $\$ 5007$ & $\$ 5008$ \\
\hline
\end{tabular}

FIGURE 6: PART RESOURCE/ATTRIBUTE DATA TABLE 


\begin{tabular}{|c|c|}
\hline location & object id \\
\hline chuck jaw & \$J005 \\
\hline gripper finger & $\$ F 003$ \\
\hline NC program & FLANGE ( 2 1/4) \\
\hline tool pos. 1 & $\$ T 003$ \\
\hline tool pos. 2 & $\$ 1007$ \\
\hline tool pos. 3 & \$T013 \\
\hline tool pos. 4 & \$T009 \\
\hline tool pos. 5 & \$T035 \\
\hline tool pos. 6 & \$T008 \\
\hline tool pos. 7 & $\$ T 030$ \\
\hline tool pos. 8 & \$T028 \\
\hline tool pos. 9 & \$T014 \\
\hline tool pos. 10 & \$T018 \\
\hline tool pos. 11 & $\$ T 012$ \\
\hline tool pos. 12 & \$T011 \\
\hline
\end{tabular}

FIGURE 7: WORKSTATION CONFIGURATION TABLE

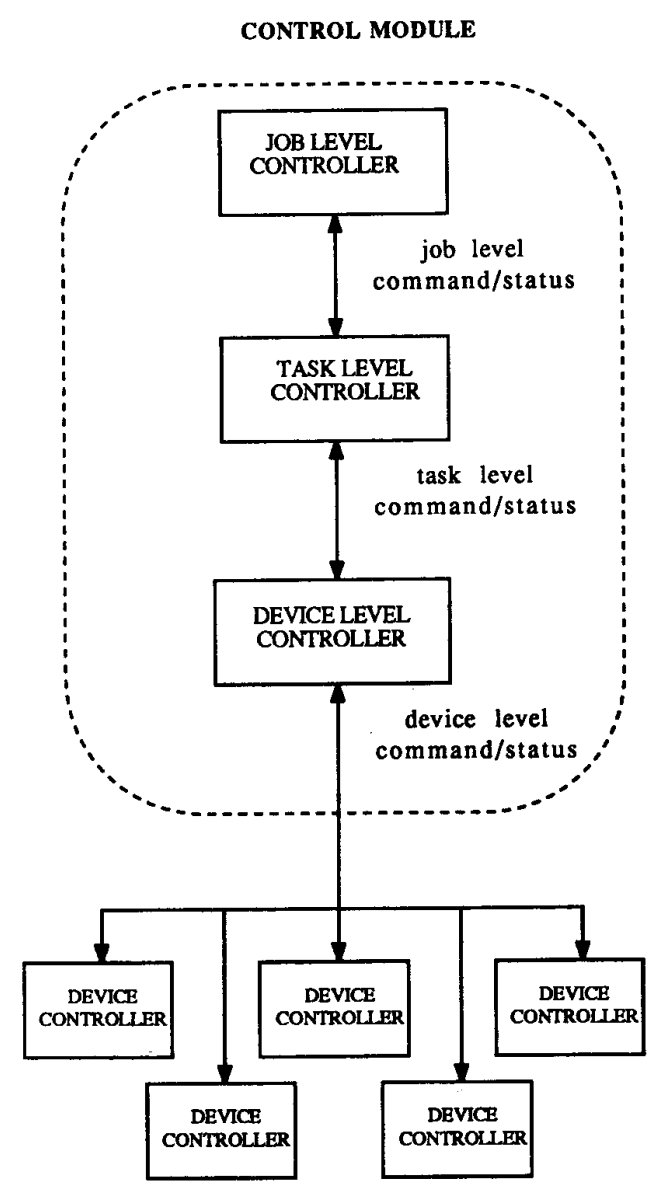

FIGURE 8: CONTROL MODULE ARCHITECTURE 